\title{
Experimental Evidence of the Real Multimode Nature of Geometric Parametric Instability
}

\author{
Y. Leventoux ${ }^{1}$, G. Granger ${ }^{1}$, A. Tonello ${ }^{1}$, K. Krupa ${ }^{2,3}$, G. Millot ${ }^{2}$, S. Wabnitz ${ }^{4}$, S. Février ${ }^{1}$, and V. Couderc ${ }^{1}$ \\ 1. Université de Limoges, XLIM, UMR CNRS 7252, 123 Avenue A. Thomas, 87060 Limoges, France \\ 2. Université Bourgogne Franche-Comté, ICB UMR CNRS 6303, 9 Avenue A. Savary, 21078 Dijon, France \\ 3. Institute of Physical Chemistry Polish Academy of Sciences, ul. Kasprzaka 44/52, 01-224 Warsaw, Poland \\ 4. DIET, Sapienza Università di Roma, Via Eudossiana 18, 00184 Roma, Italy \\ yann.leventoux@xlim.fr
}

Abstract: We show experimentally that geometric parametric instability in graded-index multimode fibers is composed by several multimode spectral components. The experimental observation is obtained by using a new 3D technique of high-resolution spatial and spectral analysis. (O) 2020 The Author(s)

\section{Introduction}

Parametric instability (PI) is a well-known phenomenon largely reported in optics, as well as in several other domains of physics [1]. In optical fibers, spectral sideband generation is generally enabled by parametric processes or by modulation instability resulting from the combined effect of Kerr nonlinearity and fiber dispersion. One can observe the generation of sidebands when starting from a strong pump in the CW or quasi-CW (long pulses) regimes when the phase matching conditions are satisfied, either in the anomalous dispersion regime [2], or in the normal dispersion in the presence of higher-order dispersion [3] or birefringence [4]. PI in optical fiber is seen in the presence of periodic modulation of the refractive index [5]. More recently, it has been demonstrated that an intense multimode beam in graded-index multimode fibers (GRIN MMFs) can create, through the Kerr effect and the self-imaging process, a longitudinal modulation of the core refractive index. Such geometric parametric instability (GPI) generates from GRIN MMFs a series of unequally spaced sidebands with large frequency shifts from the pump, and exhibiting a multimode bell-shaped transverse distribution [6]. Even if the multimode nature of sidebands is already proven by their spatial pattern, which cannot fit in a single transverse mode, the results of current literature suffer from low spectral resolutions. As a result, it has been difficult to identify the modal content of parametric sidebands.

In this work, we present a new 3D spatial and spectral technique able to identify the dominant modal content of spectral sidebands generated by the GPI process. The spatial intensity distributions in both the visible and infrared domains are precisely obtained, with a spectral resolution of $0.2 \mathrm{~nm}$. Thus, we unveil the multimode nature of GPI, and the presence of multiple satellite frequency conversions around the dominant sidebands.

\section{Experimental results and discussion}

The experiment is based on a longitudinally single mode subnanosecond laser source, delivering pulses of $740 \mathrm{ps}(\mathrm{FWHM})$ at a repetition rate of $27 \mathrm{kHz}$. The central wavelength is at $1064 \mathrm{~nm}$, with more than $50 \mathrm{~kW}$ peak power. The linearly polarized transverse Gaussian beam is coupled in a 50/125 GRIN MMF with a beam diameter of $44 \mu \mathrm{m}$ (measured at $1 / \mathrm{e}^{2}$ ). This fiber has a length of $6 \mathrm{~m}$, a core radius of $25 \mu \mathrm{m}$, a core-cladding index difference of 0.015 , and a numerical aperture of 0.2 .

For input peak powers lower than $0.5 \mathrm{~kW}$, corresponding to the linear propagation regime, a speckled beam is obtained at the fiber output. The near-field image of the pump wave at the MMF output is shown in figure 1(a). For high input peak powers (e.g., $40 \mathrm{~kW}$ ), a self-cleaning process is observed, leading a bell-shaped beam at the fiber output (figure 1(b)). The output beam pattern is composed by the fundamental mode of the fiber, surrounded by a multimode residual background. The first anti-Stokes GPI sideband is generated at $716 \mathrm{~nm}$, while the corresponding Stokes GPI sideband is generated at $2067 \mathrm{~nm}$. These dominant first-order GPI sidebands are surrounded by satellite lines exhibiting lower intensities. Additionally, stimulated Raman scattering (SRS) creates a continuum between $900 \mathrm{~nm}$ and $1600 \mathrm{~nm}$, which depletes the pump beam, and limits the GPI conversion efficiency. (See figure 1(c)).

In order to study GPI generation with high spectral and spatial dynamics, we developed a new method of mapping the spatial and spectral components of the output beam. In our method, we first magnify the fiber output, then we collect a tiny fraction of it via a single-mode optical fiber. In this way, we can obtain a "pixel" of the output beam pattern, that can be analyzed via an optical spectrum analyzer (OSA) (either Yokogawa AQ6375B at infrared wavelengths or Ando AQ6315E at pump and anti-Stokes wavelengths). The procedure is then repeated by moving the position of the single-mode fiber, in order to cover the entire image, pixel by pixel, with a fully automatized 
procedure. The use of OSAs allows for high dynamic range ( $>70 \mathrm{~dB})$, and a spectral resolution below the nanometer scale $(0.2 \mathrm{~nm}$ in our case) in the area of interest. We thus obtain a 3D matrix composed by the spectral profiles taken at all transverse spatial positions. Finally, the matrix is analyzed with a post-processing tool, so as to reconstruct, for each wavelength, the intensity profile of the output beam.

As an example of our results, we illustrate in Fig. 1(d) the transverse intensity distributions upon wavelength obtained around the Stokes sideband. The red curve corresponds to the average spectrum, obtained by integration of the energy over all pixels constituting the image of the output beam. We can see how the main peak of the sideband (at $2067 \mathrm{~nm}$ ) is carried by a $\mathrm{LP}_{02}$ mode. The closest lines surrounding the main peak, i.e., at $2085 \mathrm{~nm}$ and $2020 \mathrm{~nm}$ exhibit a combination of modes, mainly dominated by $\mathrm{LP}_{01}, \mathrm{LP}_{11}$ and by $\mathrm{LP}_{02}, \mathrm{LP}_{03}$ modes, respectively. For the largest spectral lines at $2120 \mathrm{~nm}$ and $2000 \mathrm{~nm}$, the energy is carried by $\mathrm{LP}_{01}$ and $\mathrm{LP}_{03}$ modes. Around the anti-Stokes sideband in the visible domain, Fig. 1(e) shows that only three peaks emerge from the spectrum. The main central peak is carried in most part by the fundamental mode $\mathrm{LP}_{01}$, whereas the two sidebands at $713 \mathrm{~nm}$ and $718 \mathrm{~nm}$ seem composed by the $\mathrm{LP}_{02}$ mode and a mix between $\mathrm{LP}_{01}$ and $\mathrm{LP}_{02}$ modes, respectively.

It is important to note that, in spite of the large conversion efficiency, GPI sidebands obtained in multimode GRIN fibers split into several lines, and may spread over several tens of nanometers. Such particular fine structure of the frequency conversion indicates that periodic self-imaging presents some imperfections. The fiber dispersion and diffraction, as well as random mode coupling are key parameters, which evolve significantly across the $270 \mathrm{THz}$ wide spectral range covered by the parametric conversion.

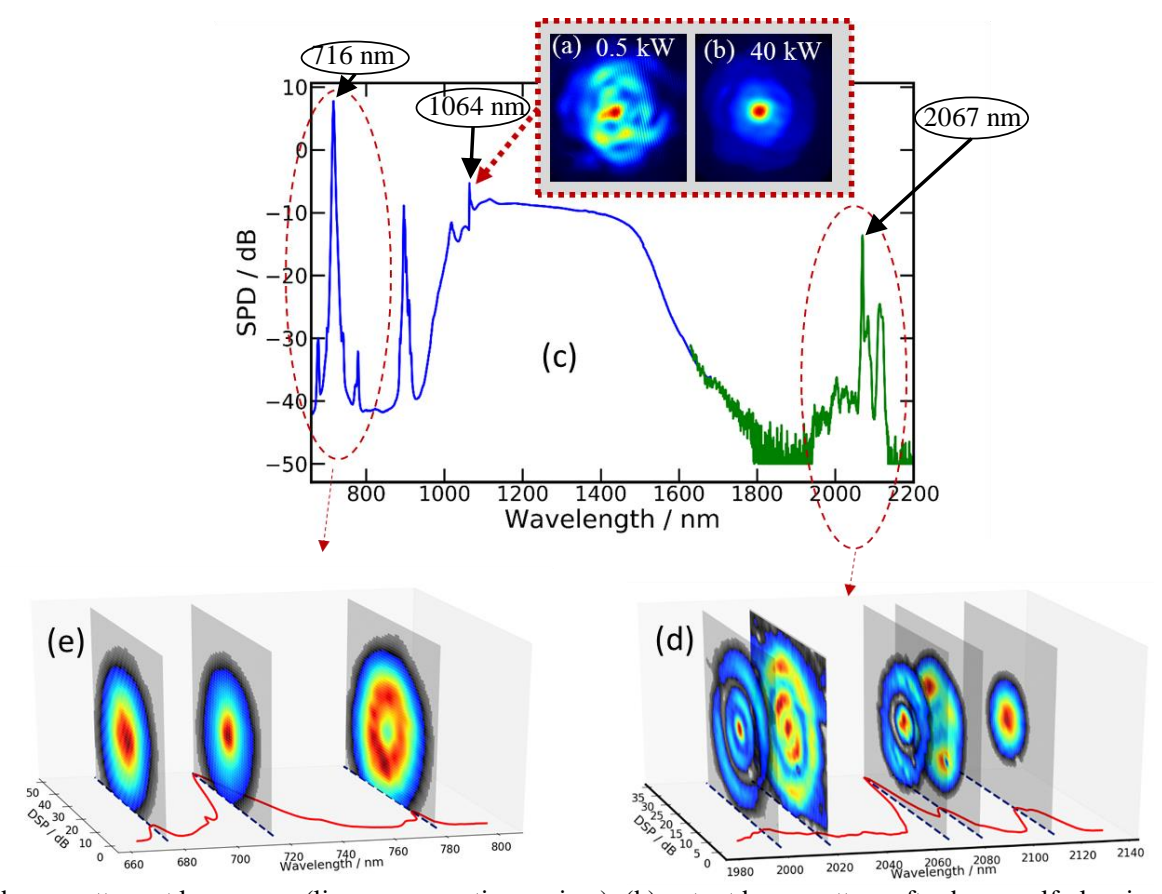

Fig. 1. (a) output beam pattern at low power (linear propagation regime); (b) output beam pattern after beam self-cleaning, (c) Output spectrum at $40 \mathrm{~kW}$, in a 6-m long 50/125 GRIN MMF; (d) transverse beam pattern associated to the GPI conversion in the infrared domain, (e): transverse

\section{Conclusion} beam pattern associated to the GPI conversion in the visible domain.

We demonstrated that geometric parametric instabilities in multimode GRIN fibers, arising from a complex nonlinear frequency mixing process of the multimode field, are composed by several spectral lines, each of them carried by different transverse modes. Such behavior may be the result of the imperfect longitudinal modulation of the refractive index, induced by Kerr nonlinearity and the beating process between modes.

[1] M. C. Cross and P. C. Hohenberg, "Pattern formation outside of equilibrium," Rev. Mod. Phys. 65, 851(1993).

[2] G. P. Agrawal, Nonlinear Fiber Optics (Academic Press, New York, 1995).

[3] S. Pitois and G. Millot, "Experimental observation of a new modulational instability spectral window induced by fourth-order dispersion in a normally dispersive single-mode optical fiber," Opt. Commun. 226, 415 (2003).

[4] S. Wabnitz, "Modulational polarization instability of light in a nonlinear birefringent dispersive medium," Phys. Rev. A 38,2018 (1988).

[5] M. Conforti, S. Trillo, A. Mussot and A. Kudlinski, "Parametric excitation of multiple resonant radiations from localized wavepackets," Sci. Rep. 5, 9433 (2015) doi:10.1038/srep09433.

[6] K. Krupa, A. Tonello, A. Barthélémy, V. Couderc, B. M. Shalaby, A. Bendahmane, G. Millot, and S. Wabnitz “Observation of Geometric Parametric Instability Induced by the Periodic Spatial Self-Imaging of Multimode Waves,” Phys. Rev. Lett. 116, 183901 (2016). 